\title{
The Experimental Technology on the Brain Impact Injuries
}

\author{
Zhiyong Yin ${ }^{1}$, Hui Zhao', Daiqin Tao ${ }^{1}$ and Shengxiong Liu ${ }^{2}$ \\ ${ }^{1}$ Chongqing Key Laboratory of Vehicle, Biological Crash Security, Department 4, Institute \\ of Surgery Research, Daping Hospital, Third Military Medical University, Chongqing \\ ${ }^{2}$ Department of Biomedical Engineering, School of Pharmacy \& Bioengineering \\ Chongqing University of Technology, Chongqing
}

China

\section{Introduction}

The traffic injury research originated during World War I. An American pilot named H. De Haven found the seat belt buckle and other cockpit parts of the fighter usually caused damage in crash accidents.Fifteen years later, he had confirmed the rubber windshield wipers could effectively prevent the metal penetrating injury caused by the forehead and facial scratches. Shortly after World War II, a mechanist J. P. Stapp led a preliminary study on the damage tolerance through accelerating pulley crash tests. Moseley (1970) tied a $5.1 \mathrm{~cm}$ thick foam rubber pad to the right chest armpit of a dog, and fixed a steel of $7.6 \mathrm{~cm}$ diameter, with a pistol firmly against the steel plate firing through the device to trigger shooting at the end of the spontaneous breathing. The model could cause the extensive parenchymal contusion, with the classical X-ray photographs performance and cardiopulmonary function changes. Anatomy of a few hours after the animal could be extensive lung hemorrhage, and there was often blood in the right bronchus.

\section{Series devices for biological impact}

In late 1960s, a simple gravity or gas-driven hammer impact animal unit has been appeared; as well as a model of liquid impact in nerve trauma study used, namely the liquid through the closed pipeline to transmit the hammer force to the brain tissue to cause damage. With the deepening of the study, it had been noted that the force changing on organisms and the precise control were critical for the study of dynamic effects. It needed to establish an ideal experimental device or model on the impact injury studies, that a series of bio-shock devices had been developed.

\section{1) Vertical biological impact machine}

1. Controllable biological impact systems

In 1974 the Swedish scholar successfully developed a controllable biological impact system to do impact injury experiments. It composed of the tower and the hit platform. The tower was equipped with a vertical pipe and cable guide. The hammer could vertically drop along the guide under the action of gravity acceleration. The impact platform included the steel 
frame, the second hammer, the second hammer stopper and animal movement supporting frames. The impact machine works by: The hammer from different heights along the guide rail whereabouts -- drove down the second hammer impact sports -- animals -- injury. It was by adjusting the height $(0.1 \sim 25 \mathrm{~m})$ and the quality $(2 \sim 10 \mathrm{~kg})$ of the hammer to get different impact velocities $(1 \sim 20 \mathrm{~m} / \mathrm{s})$ and impact energy; by adjusting the height of animal backstop and the relative position of the second hammer to change the compression degree; by replacing the second hammer head to get different impact shapes and areas. In addition, there were mechanical power sliding displacement sensors and accelerometers which could respectively recorded in three parameters of time curves for the second hammer as the motion displacement, the displacement of the body wall deformation and the impact acceleration. The force sensor on the animal backstop could record the pressure pulses delivered to the body. The hammer was of a great range of quality that it was suitable for large animal impact injury experiments, but of a lower impact velocity for the drive limit.

2. Vertical air/hydraulic biological impact machine

For different driver ways, it has derived a variety of types of vertical biological impact machines. In addition to the type of free-fall impact machine, the vertical impact machine driven by pressure has been widely used. Because of power-driven, the hammer can provide higher impact velocity without falling from high altitude, which reduced the machine size greatly. Ways includes the gas and the liquid drive that two machines are basically of the same structure and working principle. These machines are mainly formed by the gas/liquid access, the sealed high-pressure piston, the impact hammer (piston), the test equipment and animal injuries units. The piston piston cylinder is divided into upper and lower chambers. The high-pressure gas or liquid goes into the chamber, pushing the piston downward to impact to the animal. The gas or the liquid in the lower champer discharges through the pipeline. In structure, the impact hammer and the piston has been integrated. The head of the piston becomes the hitting side, and can be made shapes of the steering wheel or others as needed, in order to directly simulate the whole process of traffic injuries caused by different parts of motor vehicle. There are energy-absorbing cushions under the piston cylinder head side to prevent downward movement of the piston to adjust the shock compression rate. The device is set on the rear chamber to monitor the moving process of the piston. Sensors are put under the animal injury platform to record the pressure pulses of the loading process of the body and the body's output during the impact.

Because gas has compressibility, the high pressure gas makes piston move forward quickly at the early stage of piston movement, and then it turns into a uniform motion. While driving the piston with liquid, the piston impacts animals with constant speed. The range of impact velocity of perpendicular type gas, liquid biology is commonly between $5 \mathrm{~m} / \mathrm{s}$ and $20 \mathrm{~m} / \mathrm{s}$.

3. Biological hit machine of horizontal type

Biological hit machine of horizontal type always moves as the high pressure air drives impact hammer. In 1978, the Viano etc. in motor vehicles research lab of the United States Michigan state biological medical department used hit machine of gas driving type. The main part of machine is the bubble similar to the above-mentioned high pressure cylinder, when launching trigger device, high pressure gas in the high-pressure chamber drives piston to do high speed forward movement and hit secondary hammer which hits animals or other simulative contents. The forward movement distance of piston is $10 \mathrm{~cm}$, the weight of secondary hammer is $23.4 \mathrm{~kg}$ hung by guide ropes, the forward movement distance is $20 \mathrm{~cm}$. Compared to vertical hit machine, animal injury frame has made a big improvement; 
first, it can slide forward with fixed animal car when hitting animal; second, the pulley placed animal is hung on the framework which can slide on the rail, when hitting framework, animal and sliding framework do the reverse movement because of inertance, and it leads to damage when hitting simulative steering wheel fixed on framework.

4. Several domestic biological hit machines

The third military medical university institute of surgical field first successfully developed biological hit machine series of type BIM-I, II and III in 1990 in China, it provided a good experimental method for research of injury by collision.

\section{1) Vertical biological hit machine of type BIM-I}

This machine is composed of the impacting tower, cushion and animal injury frame with about six tons of total weight. The impacting tower is welded from angel steel, it forms closed structure with outsourcing wind sheet. The weight of impact hammer gradually increases $0.5 \mathrm{~kg}$ every time and its heaviest weight is $50 \mathrm{~kg}$, the drop height can be controlled in $0.5 \sim 36.5 \mathrm{~m}$. Impact hammer can trigger buffering devices automatically when rising to a predetermined height, and it can drop along with vertical orientation steel wire to hit secondary hammer.

Cushion consists of about a ton of table-board, pillar, buffer block and secondary hammer, its main function is to control impact range and absorb great energy produced by impact. The fastest impact speed of this machine is $26 \mathrm{~m} / \mathrm{s}$, and the instant impact speed simulated is $90 \mathrm{~km} / \mathrm{h}$.

Animal hurt frame can fix animals flexibly, according to the adjustment of horizontal and vertical position; it can precisely control impact site and the impact amplitude of the compression.

\section{2) Horizontal biological hit machine of type BIM- II}

This machine mainly consists of high-speed air cannon, secondary hammer, animal hurt frame, sliding rail system and velocity measurement system with the total weight of about two tons. The pressure of gas storage tank in high-speed gas cannon is $25 \mathrm{MPa}$, but the practical working pressure is no more than $10 \mathrm{MPa}$, the opening time of pneumatic valve is about $2 \mathrm{~ms}$, diameter and length of bore is $50 \mathrm{~mm}$ and $1300 \mathrm{~mm}$, the fastest initial speed of bore can reach to $90 \mathrm{~m} / \mathrm{s}$, it can simulate crash condition that the bus speed is no more than $320 \mathrm{~km} / \mathrm{h}$. It can accurately control impact amplitude of the compression through the secondary hammer, different impact area and geometric shape can be chosen when hitting head. Using secondary hammer seat as limiting stopper to avoid bullet breaking away from bore, and avoid the damage happened when bullet impacts with other objects, bullet can be used again. In order to adapt to research needs of different kinds of animal injury by collision, both bullet and secondary hammer have various standards of length and quality.

Velocity measurement system consists of speed measurement table, laser generator, shock tube beam splitter, guiding light implement, photosensitive components and signal amplification receiving circuit, the error of measuring secondary hammer speed is no more than $0.01 \mathrm{~mm} / \mathrm{s}$, it fully meet the requirement of speed measuring precision in the biological experiment.

Impact can be divided into standard static state and dynamic according to the difference of animal hurt frame and ways of fixation. Impact way of standard static state adopts fixed animal hurt frame, then animals cannot move forward when hitting. In the biomechanical testing experiments, it is need to put some sensors on the animal body, therefore, impact 
way of standard static state is mostly adopted. The major structure of dynamic hitting way is sliding rail system, which is composed of horizontal sliding rail, holder and pulley etc., the effective length is $3.5 \mathrm{~m}$ and height is $1.9 \mathrm{~m}$. Fixing the animal on the pulley, then using secondary hammer to impact, it can move ahead with pulley after getting impact. If installing baffle in front of sliding rail, animal can strike baffle after moving, it can lead to the animal model of acceleration injury accompanying with deceleration injury. This kind of hurting way can simulate the impacting conditions that pedestrian collides with road or other objects after hitting and throwing.

\section{3) Portable biological hit machine of type BIM-III}

The structure of this machine is simple, it is easy to install and remove with the total weight of about $11 \mathrm{~kg}$, and the volume is about $70 \times 20 \times 10 \mathrm{~cm} 3$ after remove. Its working principle is to hit animals relying on impact hammer dropping from different heights; it can carry out fixed or unfixed impacts using turning institution of animal frame. It is suitable for the impact injury research on mice, rats, and guinea pig head, chest and abdomen.

\section{Injury device of deceleration injury}

The kinetic object impacts the still experimental object to make it accelerate along with external force, and the injury happens when impacting the experimental one. Deceleration damage means the damage happened with the sudden deceleration of kinetic experimental object when it impacts the still one. Because of buffer distance, the accelerated damage is relatively light, which is often limited to stress side; while the decelerated damage is relatively heavy, the damage can happen both on stress side and offside, and the offside damage is heavier. The first purpose of developing injury model of deceleration device is to make the experiment object accept certain initial velocity, and then solve the fixed problems of experimental object. Tan Yuanfu has designed a device for craniocerebral deceleration injury research with the power provision of rubber band, and with this device, he has developed rabbit craniocerebral deceleration injury model. This device consists of turn board(A), axis of rotation $(\mathrm{O})$, elastic belt(F) and holder(B), The two-thirds of the turn board is fixed to the table through the axis of rotation, turn board can rotate $360^{\circ}$, the groove $(40$ $\mathrm{mm} \times 200 \mathrm{~mm}$ ) in the front of turn board can be put animal head. There are many $600 \mathrm{~mm}$ long elastic belts at the end of turn board. the elastic belt is flabby under horizontal position, the center of turn board front end is put on the cushion (E) to make it on the horizontal position, the $35 \mathrm{~mm}$ in diameter bump anvil(C) on the holder protrudes into groove $5 \mathrm{~mm}$. When turn board is on a vertical position, the elongate elastic belt is at a high tension state, and position of turn board is fixed through a pull hook (G). Turn board return to horizontal position with the help of elasticity after loosening pull hook, at this time the rabbit head in the groove of turn board gets craniocerebral deceleration injury because of collision with bump anvil, and the degree of damage can be adjusted through the number of elastic belts. Moments after impact, turn board rebounds because of the function of cushion, at this time, the hands need to hold turn board quickly to avoid the second damage.

Author has developed an injury device of slow injury according to the transforming principle between potential energy and kinetic energy, initial velocity of experimental subject is controlled by height of fall. This device is composed of turn board (A), animal fixed trolley (E), foundation bed $(\mathrm{G})$, chopping block(C) and holder(B)(Figure 2). Sliding guide rail is installed in fixed bases, which consists of four steel wires, the degree of 
tightness is regulated by the prestressing steel wire below foundation bed. The distance that animal fixed trolley slides along with guide rail is $7.0 \mathrm{~m}$, the height is marked with scale plate(D). The rolling bearing installed around that car can make it slide freely along with the sliding guide. The animal is fixed on trolley and its head is held by holder to make its position oppose to collision anvil. Bumper block is placed between animal fixed trolley and foundation bed, and the adjustment of deceleration coming from collision between trolley and foundation bed is based on bumper block features such as elasticity coefficient and thickness, it also can protect the trolley. Relying on the device, besides a series of rabbit cerebral injuries of deceleration injury tests, we still do the injuries of deceleration injury tests of physical model.

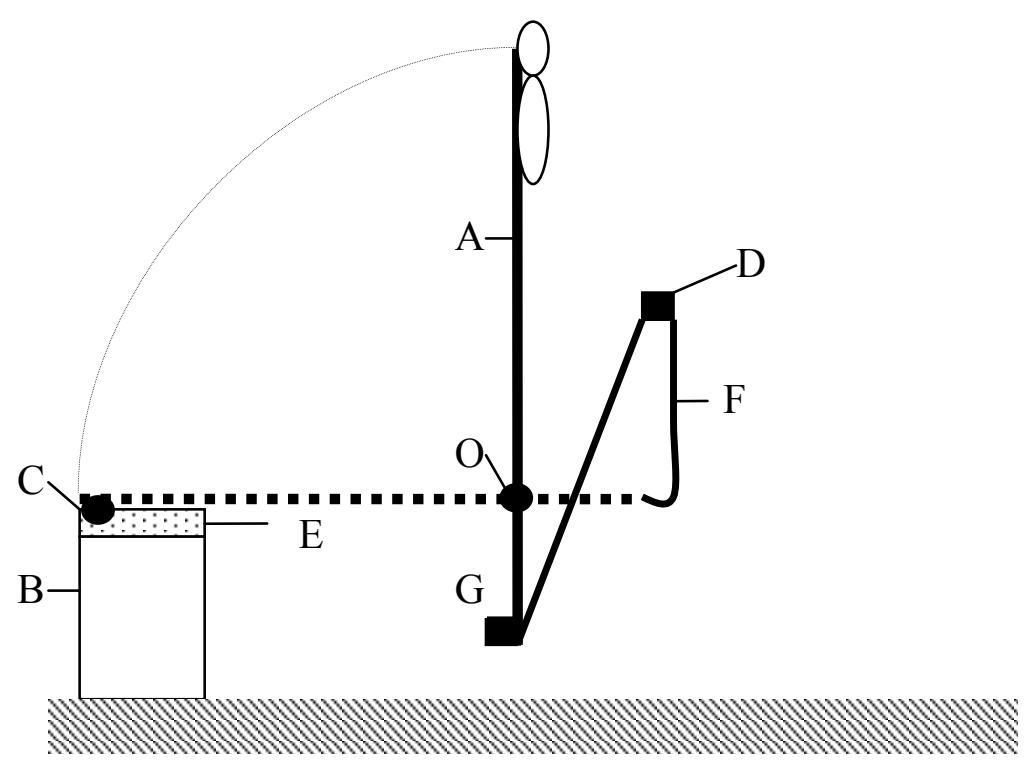

Fig. 1. Injury device of deceleration injury: A . turn board, B. holder, C. bump anvil, D. elastic belt fixed place, E. cushion, F. elastic belt, G. fixed pull hook, O. axis of rotation

\section{Collision experiment of real car}

In the experimental research of large traffic accident/injury, the foreign country regard real cars as injury tools, animals or dummy devices as received subjects, and it realistically imitates mechanical force that people get when cars collide with pedestrians, cars and other fixations, then multidimensional high-speed camera and all kinds of sensors will do detailed records and analysis. Many domestic organizations also have the condition of real car collision experiment, and have already conducted a large number of real car crash tests. The data and authenticity of real car crash tests is reliable, it is reliable mean to check and accept protective equipment and evaluate the safety standards. But the deficiency is that it needs larger venues, and cost is expensive, so it cannot be used to carry out a lot of repetitive biological experiments. 


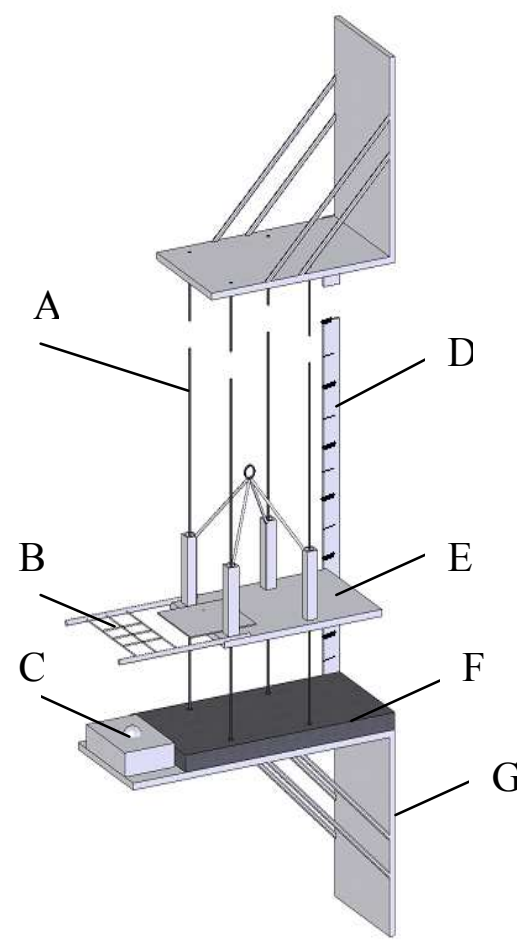

Fig. 2. Injury device of slow injury: A. guide rail, B. holder, C. chopping block, D. scale plate, E. animal fixed trolley, F. bumper block, G. foundation bed

\section{Biological impact test platform of rail type}

In fact, the occurrence of traffic injury is a dynamic process, which involves the hurting of the whole experimental objects, while the previous road traffic injury researches are mainly on the local impact tests, which cannot completely simulate the whole and dynamic engineering of traffic injury occurrence and development. Some foreign research institutes have established collision equipment for traffic medical research. And in recent years, China has also established collision laboratories in Beijing, Tianjin, Hubei and Shanghai, and they are mainly used for the real car crash tests and the test of safety performance of automobile parts; its purpose is to test the security of the car. But these researches are mainly based on engineering, and its purpose is to provide the basis and theoretical guidance for car improvement and protection of people, which combine less with medicine, especially with clinical medicine.

In view of the above reasons, the administrative office where the author is decides to establish a biological collision laboratory of track type, which gives priority to biological trolley and gives consideration to the car crash tests, its purpose is to realistically simulate occurrence process of various traffic injuries, then doo further research on trauma occurred mechanism, protection and treatment, the research laboratory began to construct in 2003, and the biological collision laboratory of track type was put into use in 2004 . 
This lab can carry out car crash test and collision experiment (including front collision, angle collision etc.); Crash conditions correspond to the basic requirements of related laws and regulations of the American FMVSS, European EEC and Chinese CMVDR294 etc, when collision speed is $\leq 64 \mathrm{~km} / \mathrm{h}$, the speed control precision is $\leq \pm 2 \%$, the maximum traction acceleration is not more than $0.5 \mathrm{~g}$, the average traction acceleration is not more than $0.3 \mathrm{~g}$; the fastest collision speed can reach to $120 \mathrm{~km} / \mathrm{h}$ 。 The biological impact test platform of rail type is composed of ground equipment, rail system, motor traction and control system, test system, lighting system and biological car etc.

\section{1) Facility}

The length of lab and acceleration road is about 88 meters and 75 meters, the width and length of collision hall is about 20 meters and 30 meters, and the total construction area is 1400 square meters. The main equipments of facility include the fixed counterguard and track beam to support collision of experimental vehicles. The counterguard is composed of reinforced concrete construction, and the impact surface is edged with a $\mathrm{T}$ shape channel steel plate, which can prevent it from movement and damage when the $2500 \mathrm{~kg}$ trolley impacts with the speed of $80 \mathrm{~km} / \mathrm{h}$. There are good vibration isolation measures between counterguard and surrounding, which can ensure that the collision experiment will not cause the vibration of the workshop and damage of surrounding ground. Rail beam is made of reinforced concrete $(78 \mathrm{~m} 2.4 \mathrm{~m} 1.0 \mathrm{~m})$, which is used to support trolley rail, traction guides and parking buffer, etc. The shape of track girder is regular to make sure that the rupture, deformation or displacement will not appear in using process. According to the test requirements, corresponding facilities can be set up in the area to complete particular test content.

\section{2) Rail system}

Rail system includes trolley rail, traction guide rail, rail cover plate, track girders, etc. Two trolley rails are installed in both sides of traction rail symmetrically; the experimental vehicles can accelerate through trolley rail or rail cover plate, and reach the setting speed in the prescribed distance, then complete collision experiment in collision hall.

1. Trolley track The trolley track has two rails of $82 \mathrm{~m}$ total length with center distance of $1.80 \mathrm{~m}$ in east-west direction. The two rails are fastened symmetrically to the track beam by the fastener with an interval of $1.25 \mathrm{~m}$.

The fine finishing heavy rail of $50 \mathrm{kgf} / \mathrm{m}$ has been welded into the seamless track. The distance between the weld and the anchor fasteners is $0.30 \mathrm{~m}$. Each rail has seven welds. The south rail is selected as the main rail while the north rail as the vice rail. The length of the main rail should be no more than $\pm 0.5 \mathrm{~mm}$. The error of the vice rail relative to the main rail in the vertical and horizontal position should be no more than $\pm 0.5 \mathrm{~mm}$. The deviation of the two tracks distance should be no more than $\pm 1 \mathrm{~mm}$. The elevation of the upper plane of the rail is $-0.010 \mathrm{~m}$ with deviation of less than $\pm 1 \mathrm{~mm}$.

2. Traction rail The traction rails are the running tracks of the traction car which is used to connect the traction rope and the experimental trolley for acceleration. The traction rails is a double structure with a small span. The track center distance is $0.235 \mathrm{~m}$. The rail is of the $25 \mathrm{~b}$ channel. The vertical and horizontal of the upper plane of the traction rails should be in level and the elevation should be no more than $\pm 2 \mathrm{~mm}$. The spacing of the edge above the guide rails is $150_{-0}^{+1} \mathrm{~mm}$ and the total length of the vertical and horizontal straight line should be no more than $\pm 2 \mathrm{~mm}$. 
3. Rail cover The rail cover is a key component both for trolley and real vehicle experiments. It Requires that when carrying out real car crash tests, it should ensure smooth movement of vehicles and the "pavement" friction coefficient should be not less than 0.75 .

\section{3) The traction motor and control system}

The traction power is from the two $225 \mathrm{kw}$ DC. The accurate closed-loop feedback control is from the DC speed controller to achieve precise control of pre-collision speed. The test data show that the motor is running smooth, with qwll synchronization of two-motor and load capability.

\section{4) The hydraulic tensioning and control system}

A mechanical tensioning device is placed in the test preparation room mainly for adjusting elastic rope seasonally. The hydraulic tensioning device is placed in the collision hall. For each test, it needs to do tension work. The hydraulic tensioning device is arranged behind the rear-wheel drive drum. After tensioning action, the wire rope is firmly pressed in the trough of the drum rope in order to pass the torque of the motor to the wire rope.

There are two frameworks with rectangular slide on the side of the rack panel. The T-slot on the base plate and the iron plate is linked by special screws which can resist to four tons of tension. The two rope tensioners moves straightly by two hydraulic cylinders, making the rope in tension or relaxation. The tension wheel assembly runs independentlyin the rectangular slide. The tensioning assembly includes a curved file block to prevent the rope relaxed state of the slide-out round of the slot. The tensioning wheel has a diameter of 400 . The cylinder of the stainless steel tube of SMC (Japan) has magnetic limit switches in the front and rear of the two extreme positions. It transmits the signal to ban the motor running in the front and rear of the two extreme positions. The hydraulic system provides a stable and adjustable pressure for the cylinder and braking system of the hydraulic tensioning device. Pumping station has a perfect control system to contact with the upper total control system.

\section{5) lighting control system with hydraulic tensioner}

For shooting the dynamic changing process of test objects in real trolley/vehicle experiments, it needs to ensure adequate lighting illumination that it can guarantee 5000lux illumination in front of $5 \mathrm{~m} \times 2 \mathrm{~m}$ range of the barrier and that it must meet the spot uniformity, light color stable, high energy efficiency, color reproduction and good requirements. The lighting system installs on the movable three-dimensional light shelves which can meet the lighting needs of any location in the collision hall with mobile stands for light adding.

\section{6) The biological trolley}

The previous bilogical impact experiments are usually on real cars with animals. But all know that the real vehicle collision tests are of high costs with poor reproducibility between different vehicles, the long preparing time as well as the space limitations for animal numbers. This shows that it is not suitable for biological impact tests by real vehicles with animals. The large number of biological traffic crash tests is conducted to the study for traffic medicine. It is necessary to develop the biological trolley on the orbital biological impact platform for replicating the traffic injury animal models. 
To do biological trolley experiments, it needs to solve the problems like the power, the animal fixing and the energy-absorbing. The traffic injury trolley for small animals has been developed based on the orbital biological impact laboratory. The biological trolley of small animal injury is an important part of the platform including the powered trolley and the animal fixable trolley. The trolley system is directly tracked by the guide rails and the wire ropes from the motor traction system in the lab. The car is free to slide on the traction rail through the pulley (Figure 3). The powered of the car is from the mechanical hook of the fastening wire ropes. When it gets the expected speed, they leave from each other to keep the constant speed. Different types of small animals can be fixed on the animal trolley for traffic injury collision experiments. Two types of trolleys can be applied as needed to simulate the different mass of vehicle collisions, and can simulate the frontal-, the side- and the rear-collision.

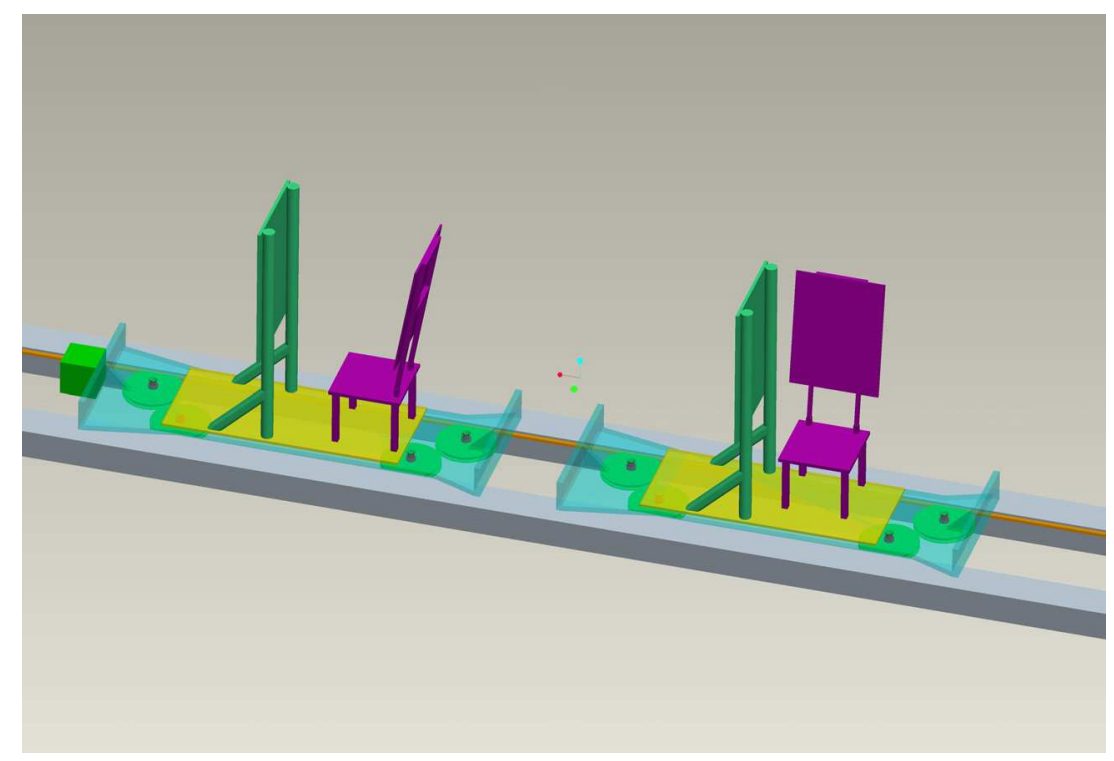

Fig. 3. The animals fixed trolley.

The energy-absorbing device is on the biological trolley or in front of the barrier. In addition to absorbing the energy of the impact moment from the barrier and the trolley, it can generate different damping forces to replicate different deceleration curves to carry out different types of traffic injury experiments for the biological trolley system. There are lots of energy absorbing forms, and usually it takes the hydraulic or combined thinwalled beams.

The structure of the hydraulic buffer is a high-pressure damping chamber. There is an oil pool above the high-pressure chamber. There are several orifices on the chamber wall as the connection of the high-pressure chamber and the oil pool. When the piston rod impacted, its front pushes the oil in to the pool. There is heat from the friction of the oil and the throttle. The majority of car's kinetic energy changses into hydraulic oil heat, and parts of the energy store in the oil pool. By adjusting the orifice area, it can get a specific deceleration waveform to meet the experimental needs. 

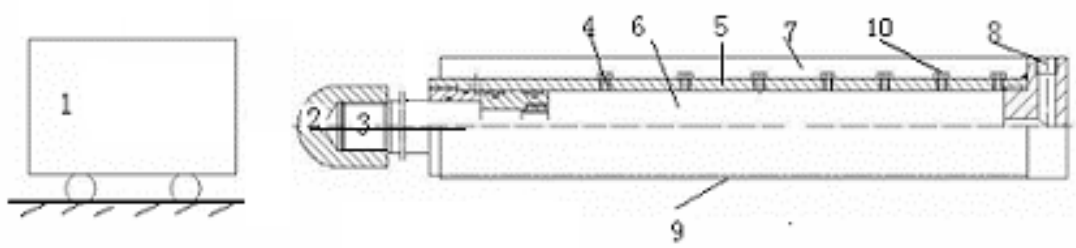

Fig. 4. The schematic diagram of the hydraulic shock absorbers: 1. trolley; 2. buffer block; 3 . rod; 4 . orifice; 5 . Inner liner; 6 . high-pressure chamber; 7. oil pool; 8 . discharge hole; 9. cylinder; 10 . cutting bolts.

The combined thin-walled beams are energy absorption devices taken a standard thin-walled square steel tube with adjusting the length and thickness of thin-walled square steel pipe combinations, to solve problems such as high costs, the single waveform and the poor reproducibility of the existing car-absorbing devices. By controlling the thin-walled square tube length of each group, this combination can control the time to make up peaks and troughs for each other in order to get a smoother deceleration waveform. The combination forms of the thin-walled square tubes are determined by the according to the mass and the deceleration curve of the trolley. The average length of thin-walled square tube is determined by the collision speed. The mounting block has been put on the trolley, so the thin-walled square tube and the trolley can be tight closely. It is applied the pre-strain in front of the thin-walled square tube with the direct rigid collision between the fixed barrier without the buffer block to let the deceleration waveform meet the test requirements. It provides a practical solution for many collision experiments on the safety of the auto parts and for biological experiments.

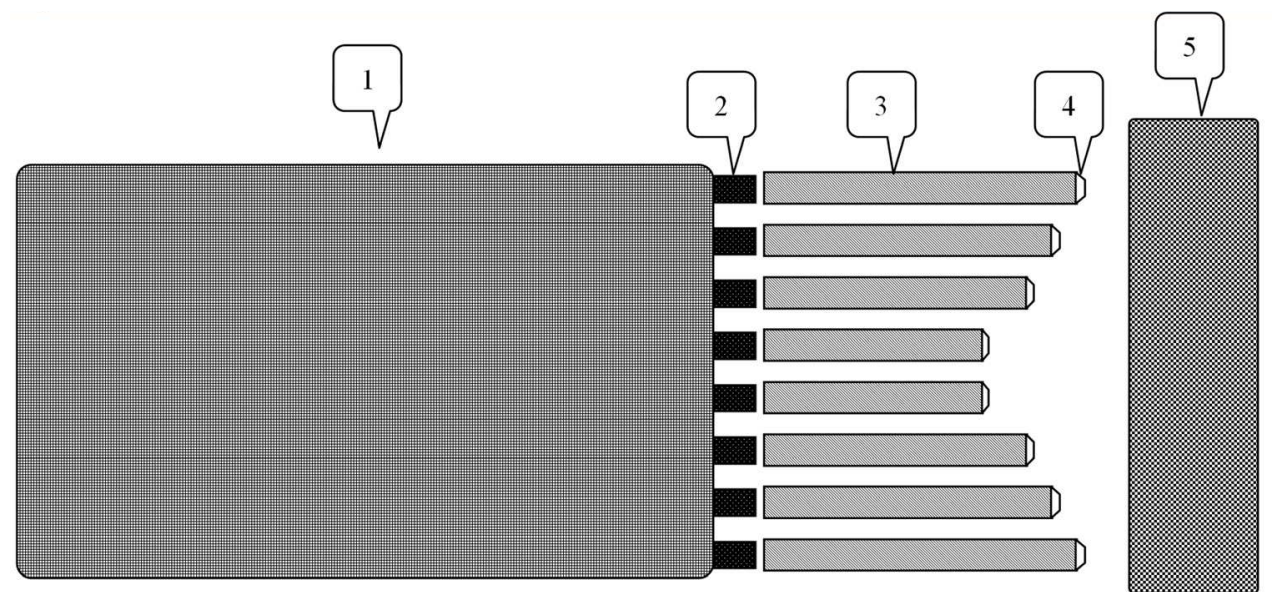

Fig. 5. The combined thin-walled beam of absorption method in trolley crash tests: 1 . Trolley; 2. Mounting Block; 3. Thin-walled square steel pipe; 4. Pre-strain; 5. Fixed barrier.

Around the car crash tests, the biological assessment of traffic safety, the vehicle crash and safety protection equipment and the security measures, it can carry out certification tests for vehicle regulations, biological collision experiments, pedestrian crash tests, rollover tests 
and motorcycle crash tests, etc. The platform can realistically simulate the impact of various vehicles and traffic injuries. It is an ideal platform for basic research of traffic injury and impact injury. It is also an important technical platform for the development of new vehicles and car parts.

\section{Computer simulation tests}

Today, computational science has been the third branch of sciences as well as the other two traditional sciences that are the theoretical science and the practical science. When the theoretical model is very complex or has not been established, or the experiment is so expensive that it cannot be done, the computer simulation experiment becomes the the main or only means. In recent years, with the development of the electronic technology, computer science and computational mathematics, it has become a trend to to study the road traffic injury through computer simulation experiments.

The traffic injury study is a wide range including the digital reconstruction of human injury in the accident scene, the biological injury testing in the laboratory and trauma biomechanics research on some individual organ damages. Since there are different purposes and objects for the traffic injury research, there are a variety of computer simulation methods and software systems which can be used to transport medical research.

\section{1) Impulse / momentum method}

Large number of real traffic accidents cases is fresh sata for traffic injury research. The traffic accident reconstruction is essential means for real case studies, and the impulse/momentum method is one of the most common means for real digital reconstructions. According to rigid body plane collision theory, the car is simplified as the plane crash model of three freedom degrees with single mass. Assuming a car driving on a flat and regular road before and after the collision, base on linear momentum and angular momentum conservation principle, it considers the energy loss by rebound factor from deformations.

PC-Crash is a typical software based on the impulse/momentum principles. In addition to the digital reconstruction for collisions like motor vehicles - motor vehicles and motor vehicles, it can also do numerical simulation for accidents like the motor vehicle - pedestrian, motor vehicle - cylinder and roll. This software can simulate up to 32 cars multi-hit at the same time and show the three-dimensional animation. A common method in the PC-Crash for accident reconstruction is taking the vehicle brake or the stop location as the optimizing target, and taking the car speed, the initial contact position, the rebound coefficient, the friction coefficient and other parameters as the optimizing variables. When the calculated results of braking traces or the stopping position consists with the real circumstances, parameters corresponding to this state are considered as closest to the real situation that it can analyse the accident cause and the human injury based on the computer simulation.

\section{2) Energy / deformation methods}

In car crashes, the deformation of the vehicle depends on the collision energy, while the collision energy depends on the relative speed between two vehicles. It shows that it is an important means of digital reconstruction by the deformation of the vehicle to infer the vehicle collision speed and to estimate the collision point. Its goal is to optimize the vehicle deformation. And the collision speed, the initial contact position, trajectory, friction coefficient and other parameters are optimization variables to do iterative calculation. When the calculation of the vehicle deformation consists with the real circumstances of the 
accident, it is considered as the closest to the real situation under the state corresponding to the parameters.

A typical software based on the energy/deformation theory is SMAC (Simulation Model of Automobile Collisions). The software is an open process. In use, the user need to develop in form of card on vehicle size, inertia, deformation and tire characteristics, as well as initial velocity, angle and motion control input, etc. These values can be modified at any time. The program calculated the detailed information such as speed, acceleration, position, angle, deformation and the force of each tire and other values by the collision process. Users modify the initial value by constantly adjusting the input of the initial velocity, impact angle and other variables according to the differences between simulation results and actual results, until the simulation results matched up with physical evidence. In recent years, MSMAC has been continuously developed. Users need only simply enter the trajectory of the collision, the vehicle deformation and the stop position that the program can optimize computing and eventually get a real situation with the closest results.

\section{3) Multi-body dynamics Method}

Currently, human biomechanics research based on numerical simulation techniques receives more and more attention in the analysis of traffic accidents. By the numerical simulation technology, it can reasonablely interpret in mechanics for the collision part of the bodies, the injury degree and causes. The body damage analysis can directly reflect the contact position and force size between people and cars, and people and ground. The accident reconstruction method based on the impulse/momentum principles or energy/deformation theory only depends on the brake marks or deformation of the body contours of the vehicle velocity and other parameters to solve problems. It cannot analyse or describe the human body injuries in the accidents.

Multi-body dynamics analysis is a powerful tool for human injury analysis. It is a mechanical branch combining the rigid body mechanics, analytical mechanics and the computer technology. Multi-body dynamics has versatility in calculating the movement of large displacement etc. with high speed in traffic injury research.

The software of MADYMO (Mathematical Dynamic Model) is based on multi-body dynamics methods. It is now one of simulation softwares for the collision damage simulation with extensive applications. In MADYMO software, it predict the injury of the occupant/pedestrian collision and the dynamic and kinetic characteristics of vehicle structures through the establishment of the appropriate model. MADYMO models are multiple rigid bodies connected by a group of different types of hinges. They usually form an open loop tree by defining the freedom degree of the hinge, binding constraint stiffness and inertia of the rigid body to get the rigid multi-body system dynamics model. The shape of the model is composed of rigid bodies such as the plane, the cylinder, the ellipsoid or other super-ellipsoid shapes, which constitute the contact surface of the multi-body model.

MADYMO software is based on theories of multi-body dynamics to generate motion equations of multi-body system. In addition to describing movement and interaction, the multi-body motion of the system has been affected by the forces from springs, dampers and the restraint system. MADYMO software can not only be used to calculate the acceleration, displacement and contact force, but also provide some body damage index calculation, such as head injury index (HIC), Severity Index, chest resultant acceleration values, chest injury index, viscosity index and femur axial load and so on. Although the original mind of MADYMO design was for the study of vehicle collision mechanics, it has been applied to 
analyze other modes of transport such as trains crash, aircraft, motorcycles and bicycles, while the software has also been used to evaluate the applicability of different restraint systems, such as seat belts and airbags.

\section{4) FEM (Finite Element Method)}

The finite element method (FEM) is a more innovative and effective numerical method developed for computer use. This method originated from aeronautical engineering in aircraft structure matrix analysis in the middle of 20th century.

For the structure matrix analysis, the overall structure can be seen as a collection interconnected by limited numbers of mechanics units, and each unit can be seen as the building brick that their assemblies can provide the overall mechanical structure properties. In 1960, an aircraft structural engineer named Clough firstly used the term of Finite Element Method in his paper to solve the plane elasticity problem. Since then, not only engineers but also mathematicians and mechanics began to realize the efficacy and the great prospect of the finite element method that they have been in-depth discussed to give it a more solid theoretical foundation. In the joint efforts of engineers and scholars, the finite element method has gone into the real continuum mechanics, becoming one of the most effective ways for solving mechanical problems. In 1969, Fridenbery finited the element theory for the first time, then it has been applied as a new methods and theories to the medical field on human biomechanics research. Currently, FEM has been widely used in the human body biomechanics and collision damage mechanics.

Typical FEM-related softwares include:

1. LS-DYNA

LS-DYNA program is mainly based on Lagrange algorithm, with ALE and Euler algorithms; oriented in the explicit solution, with the implicit solution function; on non-linear dynamic analysis with the static analysis. LS-DYNA program currently has 140 kinds of metal and non-metallic materials, with consideration of material failure, damage, viscosity, creep and strain rate associated with such properties.

2. ABAQUS

ABAQUS provides a wide range of functions, and very easy to use. Input ABAQUS data first, then enter the options block. The beginning of each option is labeled a descriptive name, so it is very convenient for the achieve of ABAQUS data. A lot of complex problems can be simulated easily by different combinations of option blocks. For example, for complex multicomponent combination of simulation problems, it is by combination of defining each option bocks of component set sizes with the corresponding material properties. For lots of simulations, even for some higher non-linear problems, users only need to provide some project data, set sizes of the structure, material properties, boundary conditions and load conditions. In some non-linear analysis, ABAQUS can automatically select the appropriate load increments and convergence accuracy. It can not only choose the right parameters, but also to adjust the parameters in the analysis process to ensure effective exact solutions. By accurate definition of parameters, users can control the numerical results effectively.

\section{References}

[1] Xiaoli Guo, Peifang Zhu, Zhengguo Wang, etc.Animal experimental models of traffic injury caused by car crash to guardrail. Chinese Journal of Clinical Rehabilitation. 2005, 9(30):120-122. 
[2] Xiaoli Guo, Zhengguo Wang, Peifang Zhu, etc.Characteristics of traffic injuries caused by lateral impact of cars in pigs.Journal of Traumatic Surgery. 2005, 7(4):251-254.

[3] Xiaoli Guo, Peifang Zhu, Zhengguo Wang, etc. Experimental study of traffic injuries caused by frontal crashes of cars. Chinese Journal of Trauma. 2005, 21(5):378-380.

[4] Xianlong Jin, Xiaoyun Zhang. 2007. Theory and practice of digital traffic accident reconstruction. Beijing: China Communications Press.

[5] Renxian Li.2004. The basis of the finite element method, 2nd edition. Beijing: National Defence Industry Press.

[6] Hui Zhao, Zhiyong Yin, Zhengguo Wang, etc.Study of traffic injury of rear crash in rabbits.Journal of Traumatic Surgery. 2006, 8(3):249-252.

[7] Hui Zhao, Zhiyong Yin, Zhengguo Wang, etc. Study on traffic injury of front crash in rabbits. Acta Academiae Medicinae Militaris Tertiae. 2007, 29(17):1657-1659.

[8] Hui Zhao, Zhiyong Yin, Rong Chen, etc. 2009.Experimental study on decelerationinduced brain injury in rabbits. Journal of Traumatic Surgery. 2009, 11(4): 306-309.

[9] Zhengguo Wang.1997. Traffic Medicine. 1st edition. Tianjin: Tianjin Science and Technology Press. 


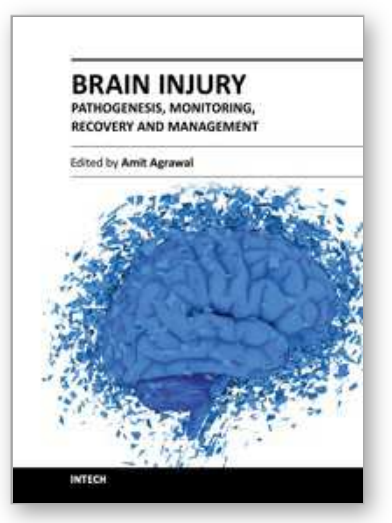

\author{
Brain Injury - Pathogenesis, Monitoring, Recovery and \\ Management \\ Edited by Prof. Amit Agrawal
}

ISBN 978-953-51-0265-6

Hard cover, 522 pages

Publisher InTech

Published online 23, March, 2012

Published in print edition March, 2012

The present two volume book "Brain Injury" is distinctive in its presentation and includes a wealth of updated information on many aspects in the field of brain injury. The Book is devoted to the pathogenesis of brain injury, concepts in cerebral blood flow and metabolism, investigative approaches and monitoring of brain injured, different protective mechanisms and recovery and management approach to these individuals, functional and endocrine aspects of brain injuries, approaches to rehabilitation of brain injured and preventive aspects of traumatic brain injuries. The collective contribution from experts in brain injury research area would be successfully conveyed to the readers and readers will find this book to be a valuable guide to further develop their understanding about brain injury.

\title{
How to reference
}

In order to correctly reference this scholarly work, feel free to copy and paste the following:

Zhiyong Yin, Hui Zhao, Daiqin Tao and Shengxiong Liu (2012). The Experimental Technology on the Brain Impact Injuries, Brain Injury - Pathogenesis, Monitoring, Recovery and Management, Prof. Amit Agrawal (Ed.), ISBN: 978-953-51-0265-6, InTech, Available from: http://www.intechopen.com/books/brain-injurypathogenesis-monitoring-recovery-and-management/the-experimental-technology-on-the-brain-impact-injuries

\section{INTECH}

open science | open minds

\section{InTech Europe}

University Campus STeP Ri

Slavka Krautzeka 83/A

51000 Rijeka, Croatia

Phone: +385 (51) 770447

Fax: +385 (51) 686166

www.intechopen.com

\section{InTech China}

Unit 405, Office Block, Hotel Equatorial Shanghai

No.65, Yan An Road (West), Shanghai, 200040, China

中国上海市延安西路65号上海国际贵都大饭店办公楼405单元

Phone: +86-21-62489820

Fax: +86-21-62489821 
(C) 2012 The Author(s). Licensee IntechOpen. This is an open access article distributed under the terms of the Creative Commons Attribution 3.0 License, which permits unrestricted use, distribution, and reproduction in any medium, provided the original work is properly cited. 